\title{
SÍNDROME METABÓLICO EN EMPLEADOS DE PANADERÍA: UN ESTUDIO PILOTO
}

METABOLIC SYNDROME IN BAKERY EMPLOYEES: A PILOT STUDY

\section{MEDINA QUEVEDO PABLO*, MARTIN DEL CAMPO NAVARRO ALICIA SARAHY*, MÁRQUEZ HERMOSILLO NOÉ**}

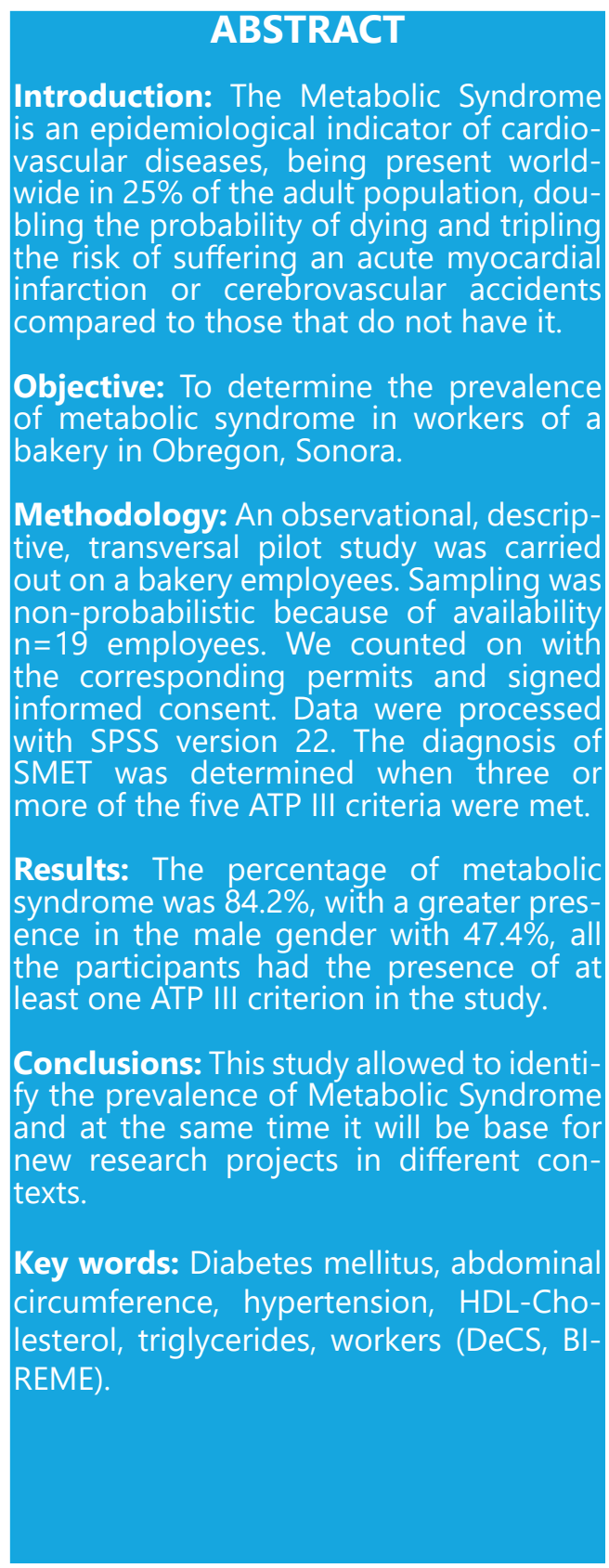

\section{RESUMEN:}

ntroducción: El Síndrome Metabólico es indicador epidemiológico de las enfermedades cardiovasculares, estando presente a nivel mundial en el 25\% de la población adulta, duplicando la probabilidad de morir y triplicando el riesgo de sufrir un infarto agudo al miocardio o accidentes cerebrovasculares comparado con los que no lo tienen.

Objetivo: Determinar la prevalencia de Síndrome Metabólico en trabajadores de una panadería de Obregón, Sonora.

Metodología: Se realizó un estudio piloto observacional, descriptivo, transversal en empleados de una panificadora. El muestreo fue no probabilístico por disponibilidad $n=19$ empleados. Se contó con los permisos correspondientes y firma de consentimiento informado. Los datos fueron procesados en SPSS versión 22. Se determinó el diagnóstico de SMET cuando se cumplieron con tres o más de los cinco criterios del ATP III.

Resultados: El porcentaje de síndrome metabólico fue del 84.2\%, con mayor presencia en el género masculino con el 47.4\%, todos los participantes tuvieron la presencia de por lo menos un criterio ATP III en el estudio.

Conclusiones: Este estudio permitió identificar la prevalencia de síndrome metabólico y a su vez será base para nuevos proyectos de investigación en diferentes contextos.

Palabras claves: Diabetes mellitus, circunferencia abdominal, hipertensión, HDL-Colesterol, triglicéridos, trabajadores (DeCS, BIREME).

*Pasante de Servicio Social de Licenciatura en Enfermería. División de Ciencias Biológicas y de la Salud. Universidad de Sonora, Campus Cajeme. Teléfono: (642)142-4858. Correo electrónico: chefpabliny@hotmail.com

**Pasante de Servicio Social de Licenciatura en Nutrición. Centro de Estudios Universitarios Vizcaya de las Américas, Campus Cd. Obregón. 


\section{INTRODUCCIÓN}

Gerald Reaven (1) en 1988 definió como síndrome X a la presencia conjunta de intolerancia a la glucosa, dislipidemia e hipertensión arterial. Más adelante se le denominó como Síndrome Metabólico (SMET) y se ha constituido como uno de los problemas sanitarios más graves del siglo XXI.

EI SMET se caracteriza por un conjunto de factores de riesgo que engloba el aumento de glucosa, triglicéridos, presión arterial, circunferencia abdominal y la disminución de HDL-Colesterol. Conforme una persona suma estos factores, el riesgo de diabetes y enfermedad cardiovascular aterosclerosa aumentan (2). Así emerge la necesidad de establecer el número de criterios presentes en un individuo, así como establecer las cifras anormales de cada uno de ellos para considerar el diagnostico de SMET.

A partir de esto varias organizaciones y grupos internacionales ${ }^{(3-7)}$ publicaron propuestas bajo sus propios criterios de diagnóstico, sin embargo algunas de ellas tenían limitaciones para su aceptación y aplicación clínica, hasta que se introdujo la definición del Adult Treatment Panel III o ATP-III por sus siglas en inglés ${ }^{(5)}$ siendo esté muy popular por su sencillez ${ }^{(8)}$ y de los más utilizados ${ }^{(9)}$ (tabla 1 ).

EI SMET es indicador epidemiológico de las enfermedades cardiovasculares, estando presente a nivel mundial en el $25 \%$ de la población adulta, duplicando la probabilidad de morir y triplicando el riesgo de sufrir infartos o

Tabla 1. Criterios de definición de Síndrome Metabólico según distintas organizaciones internacionales

\begin{tabular}{|c|c|c|c|c|c|}
\hline Criterios & $\begin{array}{l}\text { WHO } \\
1998\end{array}$ & $\begin{array}{l}\text { EGIR } \\
1999\end{array}$ & $\begin{array}{l}\text { ATP III } \\
2001\end{array}$ & $\begin{array}{l}\text { AACE } \\
2002\end{array}$ & $\begin{array}{l}\text { IDF } \\
2005\end{array}$ \\
\hline $\begin{array}{l}\text { Resistencia } \\
\text { Insulínica }\end{array}$ & $\begin{array}{c}\text { DM Tipo } 2 \\
\text { GAA } \\
\text { ITG } \\
\text { Sí GP }<110, \\
\text { Hiperinsulemia, } \\
\begin{array}{c}\text { Captación de glucosa }<25 \% \text { en } \\
\text { un clamp euglucémico }\end{array}\end{array}$ & $\begin{array}{l}\text { RI-Hiperinsulemia valores de } \\
\text { glucosa en ayunas superiores } \\
\text { en un } 25 \% \text { a los de la pobla- } \\
\text { ción no diabética }\end{array}$ & & & \\
\hline IMC & $>30 \mathrm{~kg} / \mathrm{m}^{2}$ & & & & \\
\hline Índice Cintura & $\begin{array}{l}\text { Varón }>90 \mathrm{~cm} \\
\text { Mujer }>85 \mathrm{~cm}\end{array}$ & & $\begin{array}{l}\text { Varón >102cm } \\
\text { Mujer }>88 \mathrm{~cm}\end{array}$ & $\begin{array}{l}\text { Varón }>94 \mathrm{~cm} \\
\text { Mujer }>80 \mathrm{~cm}\end{array}$ & $\begin{array}{l}\text { Varón >94cm } \\
\text { Mujer }>80 \mathrm{~cm}\end{array}$ \\
\hline Triglicéridos & $=150 \mathrm{mg} / \mathrm{dl}$ & $=180 \mathrm{mg} / \mathrm{dl}$ & $=150 \mathrm{mg} / \mathrm{dl}$ & $=150 \mathrm{mg} / \mathrm{dl}$ & $=150 \mathrm{mg} / \mathrm{dl}$ \\
\hline Col HDL & $\begin{array}{l}<35 \mathrm{mg} / \mathrm{dl} \text { varón } \\
<39 \mathrm{mg} / \mathrm{dl} \text { mujeres }\end{array}$ & $=40 \mathrm{mg} / \mathrm{dl}$ & $\begin{array}{l}=40 \mathrm{mg} / \mathrm{dl} \text { varón } \\
<50 \mathrm{mg} / \mathrm{dl} \text { mujer }\end{array}$ & $\begin{array}{l}<40 \mathrm{mg} / \text { dl varón } \\
<50 \mathrm{mg} / \text { dl mujer }\end{array}$ & $\begin{array}{l}=40 \mathrm{mg} / \mathrm{dl} \text { varón } \\
<50 \mathrm{mg} / \mathrm{dl} \text { mujer }\end{array}$ \\
\hline Presión Arterial & $\begin{array}{c}=140 / 90 \mathrm{mmHg} \text { o en tratamiento } \\
\text { médico }\end{array}$ & $\begin{array}{c}=140 / 90 \mathrm{mmHg} \text { o en trata- } \\
\text { miento médico }\end{array}$ & $\begin{array}{l}=135 / 85 \mathrm{mmHg} \text { o en } \\
\text { tratamiento médico }\end{array}$ & $=130 / 85 \mathrm{mmHg}$ & $\begin{array}{l}\mathrm{PAS}=130 \mathrm{mmHg} \\
\mathrm{PAD}=85 \mathrm{mmHg}\end{array}$ \\
\hline
\end{tabular}

Fuente: GAA: Glucosa Anormal en Ayunas; ITG: Intolerancia a la Glucosa; IMC: Índice de Masa Corporal; WHO: World Human Organization; EGIR: European Group for Study of Insulin Resistance; ATP III: Adult Treatment Panel III of The National Cholesterol Education Program (NCEP); AACE: American Association of Clinical Endocrinologist; IDF: International Diabetes Federation 
accidentes cerebrovasculares comparado con los que no lo tienen, así como de incrementar cinco veces más las probabilidades de desarrollar Diabetes Mellitus ${ }^{(10)}$.

En una investigación estadounidense sobre la prevalencia de SMET en adultos, se reportó existencia en el $25 \%$ de los hombres blancos y $21 \%$ de las mujeres blancas, los mexicano-americanos presentaron el $29 \%$ en hombres y el $33 \%$ en mujeres (11). En Venezuela, estudios recientes realizados en personas mayores de 20 años revelaron prevalencias de SMET del $25.9 \%$ en mujeres y $40 \%$ en hombres ${ }^{(12)}$.

En nuestro país cuatro de cada diez mexicanos padece SMET ${ }^{(13)}$ y según la encuesta nacional ENSANUT (14), en México hay 26 millones de personas con sobrepeso y 22 millones con obesidad. Por lo tanto la tasa de diabetes incremento de 7\% hasta 9\% del 2006 al 2012; en el 2006 había 4.3 millones de personas con Diabetes Mellitus tipo 2 y en 2012 creció a 6.4 millones. El tener una prevalencia tan elevada y el incremento de los índices de estas enfermedades hacen que el SMET sea un problema inminente en nuestro futuro próximo.

El norte de la República Mexicana presenta las prevalencias más elevadas en comparación con los estados del sur (15). Por ejemplo un estudio realizado en Monterrey arrojó prevalencias del $63.8 \%$ en pacientes de una clínica comunitaria ${ }^{(16)}$, siendo estas cifras preocupantes para el sector salud. En Sonora ENSANUT 2012 reportó cifras alarmantes en la población mayor de 20 años, 7556 pacientes bajo tratamiento de obesidad, un creciente número de diabéticos con tan solo 9794 pacientes bajo tratamiento por Diabetes Mellitus tipo 2, son 2508 los pacientes con tratamiento para Dislipidemias y las cifras arrojan 18545 con Hipertensión Arterial (HTA) siendo Sonora el primer lugar a nivel Nacional con HTA. Se reportan 2508 pacientes con SMET, de los cuales tan solo el $39.71 \%$ están bajo control ${ }^{(17)}$. A nivel local un estudio donde se midió la prevalencia del SMET en 9 sedes del país tomándose en cuenta las ATP III (5), Ciudad Obregón mostró el mayor porcentaje con el $65.75 \%{ }^{(18)}$.

"EI SMET no solo es una realidad clínica entre prácticamente todos los grupos étnicos, raciales y geográficos, constituye un problema de salud evidente, no solo por su alta prevalencia, sino también por su papel como factor de riesgo para otras enfermedades que implican una gran morbimortalidad" (11). El realizar investigaciones en grupos vulnerables como los trabajadores de la panificación, pondrá en evidencia los factores de riesgo directos y relacionados dentro de su contexto laboral, considerando lo anterior se planteó determinar la prevalencia de Síndrome Metabólico en trabajadores de una panadería de Ciudad Obregón, Sonora.

\section{METODOLOGÍA}

Se realizó un estudio piloto de tipo observacional, descriptivo, transversal, prospectivo en empleados de una panificadora ubicada en el centro de la Cd. de Obregón, Sonora. Contando dicha empresa con 6 puestos para dar servicio en los turnos matutino, vespertino y nocturno.

El tipo de muestreo fue no probabilístico por disponibilidad en el cual participaron $n=19$ empleados conformados por panaderos, cajeros, empleadas de mostrador, repartidores, personal de limpieza y administrativos del negocio.

Previamente se obtuvo la autorización de los dueños de la panadería y se programó cita con los administrativos de la empresa en la que se explicó la metodología y objetivos de la investigación. Se pidió la colaboración para convocar a los empleados a una reunión para la exposición de objetivos, lectura y explicación del consentimiento informado, así como también de exponer los beneficios de la investigación. Se incluyeron en este estudio los empleados que estuvieran dados de alta en la nómina de la empresa y que estuvieran trabajando en alguno de los tres turnos.

Después de su aceptación y firma del consentimiento informado se citó a los participantes a un laboratorio de análisis clínicos de la ciudad, para la toma de muestra sanguínea. Como indicadores bioquímicos, se solicitó glicemia y perfil lipídico, debido a las características de la investigación solo se tomó en cuenta las cifras de HDL-Colesterol y los Triglicéridos.

La evaluación antropométrica se realizó por personal especializado PLESS de Licenciatura en Nutrición el cual calculó el peso corporal a través de una báscula previamente calibrada y certificada (TANITA BC-587) con capacidad: $0-200 \mathrm{~kg}$ y la talla con un estadiómetro de 20 202 (seca213) y se determinó el índice de masa corporal (IMC) bajo los criterios establecidos por la Organización Mundial de la Salud (OMS). La circunferencia de cintura se evaluó en posición supina luego de expulsar el aire en una espiración normal a la altura de la cicatriz umbilical utilizando una cinta antropométrica ergonómica (Seca 201).

La presión arterial fue tomada con baumanómetro manual por personal calificado pasantes de Licenciatura en Enfermería, el participante estuvo en reposo previo sentado con pies plantados al suelo y espalda recta al respaldo de la silla, tomada a la altura del corazón en miembro superior izquierdo teniendo dos tomas de control en un lapso de 15 minutos de diferencia.

Para la recolección de la información se diseñó un cuestionario a manera de entrevista, la primera parte constaba de cuatro preguntas de identificación general: nombre, 
edad, género y puesto. En la segunda parte se capturaron los datos obtenidos durante el examen físico, medidas antropométricas y resultados de laboratorio. Se consideraron como variables sociodemográficas; la edad, el género y puesto en la empresa, como variables de estudio; peso, talla, estado nutricional, perímetro abdominal, glucemia basal, tensión arterial, HDL-colesterol y triglicéridos basados en los criterios del ATP III (5).

Se hizo el diagnóstico de síndrome metabólico cuando estuvieron presentes 3 o más de los factores de riesgo, de acuerdo a los criterios del ATP III (tabla 1). Circunferencia de cintura: mayor de $102 \mathrm{~cm}$ en hombres y mayor de $88 \mathrm{~cm}$ en mujeres; dislipidemia: HDL-colesterol menor de $40 \mathrm{mg} / \mathrm{dl}$ en hombres y de $50 \mathrm{mg} / \mathrm{dl}$ en mujeres; triglicéridos mayores de $150 \mathrm{mg} / \mathrm{dl}$; presión arterial mayor de $135 / 85 \mathrm{mmHg}$; y glicemia en ayunas entre $110 \mathrm{mg} / \mathrm{dl}$ y $125 \mathrm{mg} / \mathrm{dl}^{(5)}$.

La información recolectada durante la investigación se capturo en una base de datos provisional en el paquete de Windows Excel, esta base de datos se importó al software de análisis de datos estadísticos Statistical Package for the Social Sciences (SPSS) en su versión 22, en el análisis se utilizó estadística descriptiva; para variables sociodemográficas frecuencias y proporciones y para las variables de estudio medidas de tendencia central y dispersión.

La investigación se apegó a las disposiciones establecidas en el Reglamento de la ley general de Salud en Materia de Investigación para la Salud. Titulo Segundo, Capítulo I en sus Artículos; Artículo 13, Artículo 14 Fracción I, III, V, VII, y VIII, Artículo 16, Artículo 17 Fracción II, Artículo 18, Artículo 20 y Articulo $21{ }^{(19)}$. Considerando los principios éticos y atendiendo a los participantes de una manera holística respetando la confidencialidad y previo consentimiento informado.

\section{RESULTADOS}

En relación a los datos sociodemográficos el promedio de edad fue de 39.21 años $(D E=15.79)$ teniendo como mínimo 18 años y un máximo de 62 años, con un ligero predominio para el género masculino con el $52.6 \%$ y el resto para el género femenino $47.4 \%$.

En los resultados obtenidos a través de la toma de las medidas antropométricas (tabla 2) se encontró un peso promedio de $73.87 \mathrm{Kg}(D E=18.05 \mathrm{Kg})$, la estatura promedio fue de $1.64 \mathrm{~m}(D E=10 \mathrm{~cm})$ y de acuerdo a la circunferencia abdominal el promedio fue de $103.47 \mathrm{~cm}(D E=$ $16.76 \mathrm{~cm})$.

El estado nutricional determinado por IMC muestra al $31.6 \%$ de los participantes en normo peso, seguido del $26.3 \%$ con sobrepeso y con el mismo porcentaje el estado de obesidad tipo I y obesidad tipo II en el $10.5 \%$ de ellos (tabla 3), de acuerdo a las medidas de Circunferencia Abdominal y criterios de ATP III (5) se obtuvo como resultado que el $36.8 \%$ de los hombres y el $31.6 \%$ de las mujeres cumplieron con este criterio (tabla 4).

Para las cifras de glucosa en los participantes, el promedio fue de $140.26 \mathrm{mg} / \mathrm{dl}(D E=53.92 \mathrm{mg} / \mathrm{dl})$, con un valor mínimo de $95 \mathrm{mg} / \mathrm{dl}$ y máximo de $250 \mathrm{mg} / \mathrm{dl}$. Se encontró una prevalencia del $57.9 \%$ de Diabetes Mellitus en los participantes (tabla 5). En cuanto a las cifras obtenidas en la toma de Tensión Arterial el promedio para sistólica/ diastólica fue de $134 / 81 \mathrm{mmHg}(D E=25.2 / 11.2 \mathrm{mmHg})$, el valor máximo se situó en $185 / 109 \mathrm{mmHg}$ y el porcentaje de participantes con diagnóstico de Hipertensión Arterial previo fue del $57.9 \%$ (tabla 6).

Los datos señalan un promedio de $38.42 \mathrm{mg} / \mathrm{dl}$ ( $D E=$ $6.5 \mathrm{mg} / \mathrm{dl}$ ) de $\mathrm{HDL}$-colesterol, con un valor mínimo de $32 \mathrm{mg} / \mathrm{dl}$ y un valor máximo de $55 \mathrm{mg} / \mathrm{dl}$, y de acuerdo al criterio de cifras de HDL-colesterol de los ATP III se obtuvo que el $52.6 \%$ de los hombres y el $36.8 \%$ de las mujeres tienen cifras por debajo de lo normal, tan solo el $10.5 \%$ de los participantes se encuentran en rangos normales (tabla 7). También reportaron cifras altas de triglicéridos registrando un promedio de $247.68 \mathrm{mg} / \mathrm{dl}$ ( $D E$ $=44.06 \mathrm{mg} / \mathrm{dl}$ ) con un valor mínimo de $170 \mathrm{mg} / \mathrm{dl}$ y un valor máximo de $345 \mathrm{mg} / \mathrm{dl}$, y se encontró que el $100 \%$ de los participantes cumplen con el criterio de ATP III en cifras arriba de $150 \mathrm{mg} / \mathrm{dl}$, el $10.5 \%$ de los participantes se tenían en cifras de ligeramente elevados $(150 \mathrm{mg} / \mathrm{dl}$ a

Tabla 2. Medidas Antropométricas

\begin{tabular}{|l|c|c|c|c|c|c|}
\hline \multicolumn{1}{|c|}{ Variable } & $n$ & $\begin{array}{c}\text { Valor Míni- } \\
\text { mo }\end{array}$ & $\begin{array}{c}\text { Valor Máxi- } \\
\text { mo }\end{array}$ & M & Mdn & $D E$ \\
\hline Peso & 19 & 45 & 109 & 73.87 & 71.8 & 18.05 \\
\hline Talla & 19 & 1.50 & 1.82 & 1.64 & 1.65 & .10 \\
\hline Cir. Abd. & 19 & 70 & 123 & 103.47 & 108 & 16.76 \\
\hline
\end{tabular}

Fuente: Elaboración propia.

$\boldsymbol{n}=19$ 
Tabla 3. Estado nutricional de los participantes

\begin{tabular}{|l|c|c|c|c|c|c|}
\hline \multirow{2}{*}{$\begin{array}{c}\text { Estado Nutricio } \\
\text { (IMC) }\end{array}$} & \multicolumn{2}{|c|}{ Mujeres } & \multicolumn{2}{c|}{ Hombres } & \multicolumn{2}{c|}{ Total } \\
\cline { 2 - 7 } & $f$ & $\%$ & $f$ & $\%$ & $f$ & $\%$ \\
\hline $\begin{array}{l}\text { Delgadez } \\
17.00-18.49\end{array}$ & 1 & 5.3 & 0 & 0 & 1 & 5.3 \\
\hline $\begin{array}{l}\text { Normopeso } \\
18.50-24.99\end{array}$ & 3 & 15.8 & 3 & 15.8 & 6 & 31.6 \\
\hline $\begin{array}{l}\text { Sobrepeso } \\
\text { 25.00-29.99 }\end{array}$ & 2 & 10.5 & 3 & 15.8 & 5 & 26.3 \\
\hline $\begin{array}{l}\text { Obesidad I } \\
\text { 30.00-34.99 }\end{array}$ & 3 & 15.8 & 2 & 10.5 & 5 & 26.3 \\
\hline $\begin{array}{l}\text { Obesidad II } \\
\text { 35.00-39.99 }\end{array}$ & 0 & 0 & 2 & 10.5 & 2 & 10.5 \\
\hline
\end{tabular}

Fuente: Criterios OMS para Obesidad y Sobrepeso.

$\boldsymbol{n}=19$

$199 \mathrm{mg} / \mathrm{dl}$ ) y el resto de los participantes $89.5 \%$ se mostró en cifras elevadas a más de $200 \mathrm{mg} / \mathrm{dl}$ (tabla 8 ).

Dando como resultado que el porcentaje de participantes diagnosticados con Síndrome Metabólico en base a los Criterios ATP III (5) fue del $84.2 \%$, solo el $15.8 \%$ de los participantes empleados de la panadería no presentaba dicha patología en criterios acumulados, aun sin embargo todos los participantes presentó por lo menos un criterio ATP III en el estudio (tabla 9).

\section{DISCUSIÓN}

La población de estudio estuvo conformada por un total de $n=19$ de los cuales en el perfil sociodemográfico se tuvo una tendencia hacia el de género masculino con el $52.6 \%$, datos que corresponden a los indicadores de ocupación y empleo de INEGI ${ }^{(20)}$ en el cual destaca que la población económicamente activa ocupada es en su gran mayoría para el género masculino con el $61.9 \%$. La edad promedio de los sujetos de estudio fue de 39.21 años de edad ( $D E=15.79$ ) estos datos concuerdan con los reportados por INEGI (20) donde resalta que la edad promedio de la población económicamente activa es de 39.2 años de edad.

En relación con los resultados obtenidos en las medidas antropométricas se observa que el promedio para la estatura fue de $1.64 \mathrm{~m}(D E=10 \mathrm{~cm})$, el promedio de peso fue de $73.87 \mathrm{Kg}(D E=18.05 \mathrm{Kg})$ resultado relevante que refleja un exceso de $10 \mathrm{Kg}$ sobre el peso ideal en la mayoría de los participantes; en consecuencia, en el estado nutricional se observó un $63.1 \%$ en estado nutricio elevado, $26.3 \%$ con sobrepeso, $26.3 \%$ con obesidad tipo I y $10.5 \%$ con obesidad tipo II. Resultados similares a los de Palacios-Rodríguez (21) los cuales realizaron un estudio sobre la presencia de SMET en el personal de salud que trabajaba en una unidad de medicina familiar, en una población de 90 trabajadores el $75 \%$ conto con sobrepeso y obesidad, de estos mismos el $85 \%$ fue diagnosticado con SMET.

De acuerdo a los criterios de los ATP III para el diagnóstico de SMET, en las cifras de circunferencia abdominal, los participantes obtuvieron un promedio de $103.47 \mathrm{~cm}$ $(D E=16.76 \mathrm{~cm})$, resultados parecidos a los obtenidos por Granfeldt et al. ${ }^{(22)}$ quienes investigaron la capacidad predictiva de los índices antropométricos en la detección de SMET en 229 adultos de Chile, reportando un promedio de circunferencia abdominal de $98.05 \mathrm{~cm}$ $(D E=12.5 \mathrm{~cm})$.

Teniendo en cuenta las cifras establecidas de ATP III los participantes tuvieron valores por encima de lo "normal" para ambos sexos con el $68.4 \%$ de los participantes en cifras elevadas. Estos resultados coinciden a los reportados por González-Chávez y colaboradores ${ }^{(23)} \mathrm{en}$ su estudio "Factores de riesgo cardiovasculares asociados a obesidad abdominal en adultos aparentemente sanos" donde reportan que de 186 individuos estudiados el $69.9 \%$ presento obesidad abdominal y que de estos el $46.2 \%$ se diagnosticó con SMET.

En lo que respecta al criterio de glucosa basal, los participantes reportaron un promedio de $140.26 \mathrm{mg} / \mathrm{dl}$ ( $D E$ $=53.92 \mathrm{mg} / \mathrm{dl}$ ), siendo el valor mínimo $95 \mathrm{mg} / \mathrm{dl}$ y máximo de $250 \mathrm{mg} / \mathrm{dl}$, cifras elevadas tomando en cuenta el criterio de glucosa basal en los ATP III; por otra parte el $57.9 \%$ de los participantes reporto diagnóstico médico de Diabetes Mellitus, lo que constituye un alto porcentaje en la población estudiada, de acuerdo con Flores ${ }^{(13)}$ en México hay 10.3 millones de mexicanos que padecen Diabetes Mellitus tipo 2, encontrándose una prevalecía de $9.1 \%$ a $14.4 \%$ entre la población mayor de 20 años. 
Tabla 4. Participantes que cumplieron el criterio Circunferencia Abdominal de las ATP III para Síndrome Metabólico (SM)

\begin{tabular}{|l|l|l|}
\hline $\begin{array}{c}\text { Criterio ATP III Circunferencia Ab- } \\
\text { domen }\end{array}$ & $f$ & $\%$ \\
\hline Varón $>102 \mathrm{~cm}$ & 7 & 36.8 \\
\hline Mujer $>88 \mathrm{~cm}$ & 6 & 31.6 \\
\hline Normal & 6 & 31.6 \\
\hline Fuente: Criterios ATP III (2001).
\end{tabular}

Tabla 5. Participantes con diagnóstico de Diabetes

\begin{tabular}{|l|c|c|c|c|c|c|}
\hline \multirow{2}{*}{ Dx de Diabetes } & \multicolumn{3}{|c|}{ Mujeres } & \multicolumn{2}{c|}{ Hombres } & \multicolumn{2}{c|}{ Total } \\
\cline { 2 - 7 } & $f$ & $\%$ & $f$ & $\%$ & $f$ & $\%$ \\
\hline Presencia DM & 5 & 26.3 & 6 & 31.6 & 11 & 57.9 \\
\hline Ausencia DM & 4 & 21.05 & 4 & 21.05 & 8 & 42.1 \\
\hline
\end{tabular}

Nota: En base a diagnóstico previo por personal médico

$n=19$

Otro estudio realizado por Escobedo-de la peña ${ }^{(24)}$ en el que se estudiaron 1722 participantes observaron que prevalencia de Diabetes Mellitus tiene un claro aumento por la edad, pasando de $3 \%$ en el grupo de edad más joven (25 a 34 años), a 22\% en los de 55 a 64 años de edad, lo que responde a la alta prevalencia de Diabetes Mellitus en la probación estudiada.

En cuanto al criterio ATP III para la tensión arterial, la cifra promedio para Sistólica/Diastólica fue de 134/81 $\mathrm{mmHg}$ ( $D E=25.2 / 11.2 \mathrm{mmHg}$ ) encontrándose en un valor límite del normal de acuerdo a los parámetros y se menciona que para validar este criterio, las cifras de tensión arterial tendrían que ser mayor o igual a $135 / 85 \mathrm{mmHg}$, estar en tratamiento farmacológico para su control o diagnóstico médico de hipertensión. El porcentaje de participantes con diagnóstico de hipertensión fue del $57.9 \%$, porcentaje elevado en comparación con el estudio realizado por Campos-Nonato ${ }^{(25)}$ en el cual reporta en la República Mexicana una prevalencia de hipertensión del 31.5\%, sin embargo señalan que existieron diferencias significativas entre las regiones siendo $34.3 \%$ más alta en el norte del país.

Los resultados de laboratorio para las cifras de HDL-colesterol registró un promedio de $38.42 \mathrm{mg} / \mathrm{dl}(D E=$ $6.5 \mathrm{mg} / \mathrm{dl}$ ) valores por debajo de lo "normal" en ambos sexos y de acuerdo al criterio de cifras de HDL-colesterol de los ATP III, el $89.4 \%$ estuvo por debajo del rango normal siendo el $52.6 \%$ hombres y el $36.8 \%$ mujeres. Resultados similares a los reportados por Pinzón y colaboradores ${ }^{(10)}$ los que reportaron cifras de HDL-colesterol bajo tomando valores de referencia $(<40 \mathrm{mg} / \mathrm{dl}$ en varones $y<50 \mathrm{mg} / \mathrm{dl}$ en mujeres) correspondientes a los ATP III y teniendo una muestra de 155 participantes en la cual el $96.1 \%$ de ellos mostro HDL-colesterol bajo, siendo el $44.5 \%$ mujeres y el $51.6 \%$ hombres.

Para las cifras de triglicéridos se registró un promedio de $247.68 \mathrm{mg} / \mathrm{dl}(D E=44.06 \mathrm{mg} / \mathrm{dl})$ con un valor mínimo de $170 \mathrm{mg} / \mathrm{dl}$ y un valor máximo de $345 \mathrm{mg} / \mathrm{dl}$, valores superiores a los parámetros normales de acuerdo a lo estipulado en los ATP III, el 100\% de los participantes cumplen este criterio, de los cuales tan solo el $10.5 \%$ se encontró en cifras ligeramente elevadas $(150 \mathrm{mg} / \mathrm{dl}$ a $199 \mathrm{mg} / \mathrm{dl}$ ) y el resto $89.5 \%$ se encontró en cifras elevadas (más de $200 \mathrm{mg} / \mathrm{dl}$ ) teniendo un mayor porcentaje para el sexo masculino con el $52.64 \%$. Resultados parecidos a los reportados por Escobedo-de la peña ${ }^{(24)}$ que estudiaron una población de 1732 participantes reportando una prevalencia de hipertrigliceridemia mayor en hombres con el $43.3 \%$.

Por último, se determinó el diagnóstico de SMET en los empleados de la panificadora que cumplieron con tres o más de los cinco criterios para diagnóstico clínico del ATP-III ${ }^{(5)}$. De los 19 empleados el porcentaje de SMET fue del $84.2 \%$, con mayor presencia en el género masculino con el $47.4 \%$ y $36.8 \%$ para el género femenino; solo el $15.8 \%$ de los participantes no presentó SMET en criterios acumulados, sin embargo, cabe destacar que todos los participantes tuvieron la presencia de por lo menos un criterio ATP III en el estudio.

La cantidad de tres criterios se presentó en $26.3 \%$, la cantidad cuatro criterios estuvo en el $21 \%$, por último los 
Tabla 6. Participantes con diagnóstico de Hipertensión Arterial (HTA)

\begin{tabular}{|l|c|c|c|c|c|c|}
\hline \multirow{2}{*}{ Dx de HTA } & \multicolumn{2}{|c|}{ Mujeres } & \multicolumn{2}{c|}{ Hombres } & \multicolumn{2}{c|}{ Total } \\
\cline { 2 - 7 } & $\boldsymbol{f}$ & $\%$ & $\boldsymbol{f}$ & $\%$ & $\boldsymbol{f}$ & $\%$ \\
\hline $\begin{array}{l}\text { Presencia de } \\
\text { HTA }\end{array}$ & 5 & 26.3 & 6 & 31.6 & 11 & 57.9 \\
\hline $\begin{array}{l}\text { Ausencia de } \\
\text { HTA }\end{array}$ & 4 & 21.05 & 4 & 21.05 & 8 & 42.1 \\
\hline
\end{tabular}

Tabla 7. Participantes que cumplieron el criterio Colesterol HDL de las ATP III para SM

\begin{tabular}{|l|c|c|}
\hline \multicolumn{1}{|c|}{$\begin{array}{c}\text { Criterio ATP III } \\
\text { Colesterol HDL }\end{array}$} & $\boldsymbol{f}$ & \% \\
\hline Varón $=40 \mathrm{mg} / \mathrm{dl}$ & 10 & 52.6 \\
\hline Mujer $<50 \mathrm{mg} / \mathrm{dl}$ & 7 & 36.8 \\
\hline Normal & 2 & 10.5 \\
\hline
\end{tabular}

Fuente: Criterios ATP III (2001).

Tabla 8. Participantes que cumplieron el criterio Triglicéridos de las ATP III para SM

\begin{tabular}{|l|c|c|c|c|c|c|}
\hline \multicolumn{1}{|c|}{$\begin{array}{c}\text { Criterio } \\
\text { ATP III } \\
\text { Triglicéridos }\end{array}$} & \multicolumn{2}{|c|}{ Mujeres } & \multicolumn{2}{c|}{ Hombres } & \multicolumn{2}{c|}{ Total } \\
\cline { 2 - 7 } & $\boldsymbol{f}$ & $\%$ & $\boldsymbol{f}$ & $\%$ & $\boldsymbol{f}$ & $\%$ \\
\hline $\begin{array}{l}\text { Levemente Eleva- } \\
\text { De 150 a 199 }\end{array}$ & 2 & 10.5 & 0 & 0 & 2 & 10.5 \\
\hline $\begin{array}{l}\text { Elevados } \\
\text { De 200 a 499 }\end{array}$ & 7 & 36.9 & 10 & 52.6 & 17 & 89.5 \\
\hline
\end{tabular}

Fuente: Criterios ATP III (2001).

Tabla 9. Participantes con Diagnóstico de SM de acuerdo a criterios de ATP III y número de criterios presentes por sexo

\begin{tabular}{|l|c|c|c|c|c|c|}
\hline \multirow{2}{*}{$\begin{array}{c}\text { Según } \\
\text { ATP III }\end{array}$} & \multicolumn{2}{c|}{ Mujeres } & \multicolumn{2}{c|}{ Hombres } & \multicolumn{2}{c|}{ Total } \\
\cline { 2 - 7 } & $\boldsymbol{f}$ & $\boldsymbol{\%}$ & $\boldsymbol{f}$ & $\boldsymbol{\%}$ & $\boldsymbol{f}$ & $\%$ \\
\hline Dx SM & & & & & & \\
Sí & 7 & 36.8 & 9 & 47.4 & 16 & 84.2 \\
No & 2 & 10.5 & 1 & 5.3 & 3 & 15.8 \\
\hline No. Criterios & & & & & & \\
1 & 1 & 5.3 & 0 & 0 & 1 & 5.3 \\
2 & 1 & 5.25 & 1 & 5.25 & 2 & 10.5 \\
3 & 15.8 & 2 & 10.5 & 5 & 26.3 \\
4 & 0 & 0 & 4 & 21.1 & 4 & 21.1 \\
5 & 4 & 21.1 & 3 & 15.7 & 7 & 36.8 \\
\hline
\end{tabular}

Fuente: Elaboración en base a ATP III (2001). 
cinco criterios estuvo presente en el $36.9 \%$ siendo el número de criterios con mayor prevalencia. Resultados menores a los obtenidos por Valdez ${ }^{(26)}$ en el cual estudiaron 1005 participantes tomando en cuenta los criterios de los ATP III, y en el cual se determinó el diagnóstico de SMET en el $66.6 \%$ de la población, con una presencia significativa en el género femenino del $64.9 \%$ a comparación del género masculino correspondiendo el $35.1 \%$ de los participantes. Por otro lado Muñoz ${ }^{(27)}$ estudio a 329 pacientes con Diabetes Mellitus tipo 2, encontró SMET en el $77.7 \%$ de las mujeres y el $76.9 \%$ de los hombres, de acuerdo a los ATP III.

\section{CONCLUSIÓN}

De acuerdo a los resultados obtenidos existe una alta prevalencia de Síndrome Metabólico en más del $80 \%$ de la población estudiada, destacando la presencia de más de un criterio ATP III para el diagnóstico del mismo en el $100 \%$ de los participantes. Esto es un tema alarmante para el sector salud, no es de extrañar tener en nuestro estado los primeros lugares en obesidad, diabetes e hipertensión, por consecuencia las cifras de SMET son elevadas.

Es urgente implementar medidas de acción enfocadas a la prevención de cada uno de los criterios presentes en el Síndrome Metabólico, de esta manera se podrán reducir las altas prevalencias presentes en nuestra comunidad disminuyendo el riesgo cardiovascular y la tendencia a padecer diabetes mellitus. Este estudio nos permitió identificar la prevalencia de Síndrome Metabólico y a su vez será base para nuevos proyectos de investigación en diferentes contextos.

\section{REFERENCIAS BIBLIOGRÁFICAS}

1. Reaven GM. Banting lecture 1988. Role of insulin resistance in human disease. Diabetes. 1988; 37: 1595-607.

2. Wacher-Rodarte N. Epidemiologia del síndrome metabolico. Gac Méd Méx. 2009: 14(5); 384-91.

3. Alberti KG, Zimmet PZ. Definition, diagnosis and classification of diabetes mellitus and its complications. Part 1: diagnosis and classification of diabetes mellitus provisional report of a WHO consultation. Diabet Med. 1998; 15: 539-5

4. Balkau B, Charles MA. Comment on the provisional report from the WHO consultation. European Group for the study of insulin resistance (EGIR). Diabet Med. 1999; 16: $442-43$.

5. Expert Panel on Detection, Evaluation and Treatment of High Blood Cholesterol in Adults. Executive summary of the Third Report of the National Cholesterol Education Program (NCEP) Expert Panel Detection, Evaluation and
Treatment of High Blood Cholesterol in Adults (Adult Treatment Panel III). JAMA. 2001; 285: 2486-97.

6. Einhorn D, Reaven GM, Cobin RH et al. American College of Endocrinology position statement on the insulin resistance syndrome. Endocr Pract. 2003; 9: 23752.

7. Alberti KG, Zimmet P, Shaw J. The metabolic syndrome-a new worldwide definition. Lancet. 2005; 366: 1059-62.

8. Fonte N, Sanabría JG, y Bencomo LM. Factores de riesgo asociados y prevalencia de síndrome metabólico en la tercera edad. Rev de Cien Méd de Pinar del Río. 2014; 18(6): 963-73

9. Cruz López M. Sindrome Metabólico. Epidemiología del Síndrome Metabólico. Bioquimia. 2009: 34(1).

10. Pinzón A, Olimpo Velandia O, Ortiz CA, et al. Síndrome metabólico en trabajadores de un hospital de nivel III de atención Estudio SIMETRA. Acta Médica Colombiana. 2014; 39(4): 237-335.

11. Castelo Elías-Calles L, Arnold Domínguez Y, et al. Epidemiología y prevención de síndrome metabólico. Revista Cubana de Higiene y Epidemiología. 2011; 50(2): 250-6.

12. Palacios $A$, Durán $M$, y Obregón O. Factores de riesgo para el desarrollo de Diabetes Tipo 2 y Síndrome Metabólico. Revista Venezolana de Endocrinología y Metabolismo. 2012; 10(1): 34-40. Recuperado de http://www.redalyc.org/ articulo.oa?id=375540232006

13. Flores-Sáenz E. El síndrome metabólico, un problema de salud pública tan grave como el cambio climático. Boletines UAM. 2015. Recuperado de: http://www.comunicacionsocial.uam.mx/boletinesuam/050-15.html

14. Encuesta Nacional de Salud y Nutrición (ENSANUT) 2012. 2012. Recuperado de: http://ensanut.insp.mx/informes/ENSANUT2012ResultadosNacionales.pdf

15. Ramirez-Vargas E, Arnaud-Viñas MR, Delisle H. Prevalence of the metabolic syndrome and associated lifestyles in adult males from Oaxaca, Mexico. Salud Publica Méx. 2007; 49(2): 94-102.

16. Solís-Olivares CA, y Solís-Soto JM. Obesidad central en el síndrome metabólico: ¿Criterio esencial u opcional? Análisis de su efecto en pacientes con cardiopatía isquémica. Rev Mex Cardiol. 2010; 21(1): 9-18.

17. Campillo García JB. Estrategia SOD Sonora. Secretaria de Gobernación. 2015 Recuperado de: https://www.gob. $\mathrm{mx} /$ salud/documentos/estrategia-sod-sonora

18. Andrade S.A.I, \& Montsalve M.C.R. Diabetes mellitus actualizaciones, Islas Andrade: Editorial Alfil. 2013.

19. Secretaria de Salud. Ley General de Salud en Materia de Investigacion para la Salud. Diario Oficial de la Federacion. 2014. Recuperado de: http://www.dof.gob.mx/nota_detalle. php?codigo $=5339162 \&$ fecha $=02 / 04 / 2014$

20. Instituto Nacional de Estadística y Geografía (INEGI). 
Indicadores de ocupación y empleo al segundo trimestre de 2017. 2017. Recuperado de: http://www3.inegi.org.mx/ sistemas/temas $/$ default.aspx?s $=$ est $\& c=25433 \& \mathrm{t}=1$

21. Palacios-Rodriguez $R$, et al. Síndrome Metabólico en personal de la salud de una unidad de medicina familiar. Rev Med Inst Mex Seguro Soc. 2010; 48(3): 297-302.

22. Granfeldt Molina G, Ibarra Pezo J, Mosso Corral C, Muñoz Reyes S, Sáez Carrillo K \& Zapata Fuentes D. Capacidad predictiva delos índices antropométricos en la detección de Síndrome Metabólico en adultos chilenos. Archivos Latinoamericanos de Nutrición. 2015; 65(3): 152-157. Recuperado de: http://www.scielo.org.ve/scielo.php?script=sci_arttext\&pid=\$000406222015000300003\&lng=es\&tlng=es.

23. González-Chávez A, Amancio-Chassin $O$, Islas-Andrade S, Revilla-Monsalve C, Hernández-Q M, Lara-Esqueda A, et al. Factores de riesgo cardiovascular asociados a obesidad abdominal en adultos aparentemente sanos. Rev Med Inst Mex Seguro Soc. 2008; 46(3): 273-9.

24. Escobedo-de la peña J, de Jesús-Pérez R, Schargrodsky $\mathrm{H}$, y Champagne B. Prevalencia de dislipidemias en la ciudad de México y asociación con otros factores de riesgo cardiovascular. Resultados del estudio CARMELA. Gaceta Médica de México. 2014; 150: 128-36.

25. Campos-Nonato I, Hernández-Barrera L, Rojas-Martínez R, Pedroza-Tobías A, Medina García C, y Barquera S. Hipertensión arterial: prevalencia, diagnóstico oportuno, control y tendencias en adultos mexicanos. Salud Pública de México. 2013; 55.

26. Valdés Ramos E, y Bencosme Rodríguez N. Metabolic syndrome and cardiovascular disease in type 2 diabetes mellitus patients. Revista Cubana de Endocrinología. 2013; 24(2): 125-35.

27. Muñoz Batista MJ, Peña Borrego M, Hernández Gárciga FF, Pérez Lemus JF, Soler Tejera I, y Just Matos CC. Síndrome metabólico en pacientes diabéticos tipo 2 pertenecientes al área de salud Guanabo. Rev Cubana Med Gen. 2010; 26(2). 